\title{
VARIANTE DE UN SISTEMA NO HIPERBÓLICO
}

\section{Teodoro Sulca Paredes ${ }^{1}$}

Resumen: En el artículo [5] se consideró el sistema no hiperbólico

$$
\left\{\begin{array}{l}
\frac{d x}{d t}=a x+b y+P_{2}(x, y) \\
\frac{d y}{d t}=c x+d y+Q_{2}(x, y)
\end{array}\right.
$$

el cual se transforma en

$$
\left\{\begin{array}{l}
\frac{d x}{d t}=y, \\
\frac{d y}{d t}=a_{k} x^{k}[1+h(x)]+b_{n} x^{n} y[1+g(x)]+y^{2} f(x, y),
\end{array}\right.
$$

y se analizó el caso para cuando $k$ es impar. En el presente artículo analizaremos el comportamiento de las trayectorias en la vecindad de un punto singular aislado cuando $k$ es par.

Palabras Claves: Trayectorias, explosiones, estructuras topológicas, sistemas analíticos.

\section{Variant of a not Hiperbolic Systems}

Abstract: Already in the article [5] we consider the not hiperbolic system

$$
\left\{\begin{array}{l}
\frac{d x}{d t}=a x+b y+P_{2}(x, y) \\
\frac{d y}{d t}=c x+d y+Q_{2}(x, y)
\end{array}\right.
$$

which is transformed in

$$
\left\{\begin{array}{l}
\frac{d x}{d t}=y \\
\frac{d y}{d t}=a_{k} x^{k}[1+h(x)]+b_{n} x^{n} y[1+g(x)]+y^{2} f(x, y),
\end{array}\right.
$$

and analyze the case when $k$ is an odd number. In the present article will analyze the behaviour of the trajectories in the vicinity of a isolated singular point, when $k$ is a number pair.

Keywords: Trajectories, explosions, topological estructures, analytical systems.

\section{Preliminares}

Ya en el artículo [5] se consideró el sistema analítico

$$
\left\{\begin{array}{l}
\frac{d x}{d t}=a x+b y+P_{2}(x, y) \\
\frac{d y}{d t}=c x+d y+Q_{2}(x, y)
\end{array}\right.
$$

\footnotetext{
${ }^{1}$ UNMSM, Facultad de Ciencias Matemáticas. e-mail: teosulcap@hotmail.com
} 
bajo las condiciones

$$
\begin{aligned}
& |a|+|b|+|c|+|d| \neq 0 \\
& \sigma=a+d=0 \\
& \Delta=\left|\begin{array}{ll}
a & b \\
c & d
\end{array}\right|=a d-b c=0,
\end{aligned}
$$

esto es, los dos autovalores de la matriz $\left(\begin{array}{ll}a & b \\ c & d\end{array}\right)$ son iguales a cero, siendo $O(0,0)$ un punto singular aislado, $P_{2}(x, y), Q_{2}(x, y)$ analíticos en una vecindad de $O(0,0)$.

Además la ecuación (34) se transforma en

$$
\left\{\begin{array}{l}
\frac{d x}{d t}=y, \\
\frac{d y}{d t}=a_{k} x^{k}[1+h(x)]+b_{n} x^{n} y[1+g(x)]+y^{2} f(x, y)
\end{array}\right.
$$

donde $h(x), g(x)$ y $f(x, y)$ son analíticas en la vecindad $O(0,0), h(0)=g(0) ; k \geq 2, a_{k} \neq 0$. Aplicando la transformación

$$
x=x, y=\eta_{1} x \quad \cdots\left(F_{0}\right)
$$

el cual transforma $\left(A_{0}\right)$ en

$$
\left\{\begin{array}{l}
\frac{d x}{d t}=\eta_{1} x, \\
\frac{d \eta_{1}}{d t}=-\eta_{1}^{2}+a_{k} x^{k-1}[1+h(x)]+b_{n} x^{n} \eta_{1}[1+g(x)]+\eta_{1}^{2} x f\left(x, \eta_{1} x\right),
\end{array} \cdots\left(A_{1}\right)\right.
$$

luego aplicamos la transformación

$$
x=x, \eta_{1}=\eta_{2} x \quad, ; d \tau=x d t
$$

(por simplicidad notacional usaremos $t$ para denotar $\tau$ ) el cual transforma $\left(A_{1}\right)$ en

$$
\left\{\begin{array}{l}
\frac{d x}{d t}=\eta_{2} x \\
\frac{d \eta_{2}}{d t}=-2 \eta_{2}^{2}+a_{k} x^{k-3}[1+h(x)]+b_{n} x^{n-1} \eta_{2}[1+g(x)]+\eta_{2}^{2} x f\left(x, \eta_{2} x^{2}\right),
\end{array}\right.
$$

continuando de la misma forma aplicamos las transformaciones $\left(F_{0}\right),\left(F_{1}\right), \ldots,\left(F_{r-1}\right)$ donde

$$
x=x, \eta_{r-1}=\eta_{r} x \quad, d \tau_{r-1}=x d t
$$

y con lo cual $\left(A_{0}\right)$ se transforma en

$$
\left\{\begin{array}{l}
\frac{d x}{d t}=\eta_{r} x \\
\frac{d \eta_{r}}{d t}=-r \eta_{r}^{2}+a_{k} x^{k-2 r+1}[1+h(x)]+b_{n} x^{n-r+1} \eta_{r}[1+g(x)]+\eta_{r}^{2} x f\left(x, \eta_{r} x^{r}\right) .
\end{array}\right.
$$

Las transformaciones $\left(F_{1}\right), \ldots,\left(F_{r-1}\right)$ son distintas de $\left(F_{0}\right)$ en el sentido de que también hacemos una transformación en la variable temporal, tales transformaciones $F_{i}$, se conocen como explosiones. La propiedad de la transformación $\left(F_{r-1}\right)$ es que descompone estructuras topológicas complicadas de los sistemas $\left(A_{k-1}\right)$ en estructuras topológicas mas simples de puntos singulares de los sistemas $\left(A_{k}\right), k=0,1,2, \ldots$ Se continúa de la misma forma hasta el $r$-ésimo paso, cuando todos los puntos singulares de $\left(A_{k}\right)$ son simples y luego de las propiedades de la transformación inversa $F_{k}^{-1}, k=0,1,2, \ldots, r-1$ hallamos la estructura topológica del punto singular $O$ para el sistema $(A)$.

Nos referiremos a los siguientes lemas, cuyas pruebas se pueden encontrar en [1], [3] . 
Lema 1. Se tiene que

i) $\mathrm{Si} \widetilde{v}_{1}$ y $\widetilde{v}_{2}$ son sectores parabólicos del punto singular $\widetilde{O}$, entonces $w^{*}$ es la unión de una región eliptica y dos sectores parabólicos.

ii) Si $\widetilde{v}_{1}$ y $\widetilde{v}_{2}$ son regiones hiperbólicas, entonces $w^{*}$ es una región hiperbólica.

iii) Si una de las regiones $\widetilde{v}_{1}, \widetilde{v}_{2}$ es hiperbólica y la otra parabólica, entonces $w^{*}$ es una región parabólica.

Lema 2. Sea $\widetilde{O}_{1}\left(0, k_{1}\right)$ un punto silla simple del sistema

$$
\left\{\begin{array}{l}
\frac{d x}{d t}=f(x, \eta x) \\
\frac{d \eta}{d t}=\frac{g(x, \eta x)}{x^{2}}-\eta \frac{f(x, \eta x)}{x}
\end{array}\right.
$$

entonces

i) Si el punto singular $\widetilde{O}_{2}\left(0, k_{2}\right)$ es un nodo, la vecindad del punto singular $O(0,0)$ es la unión de dos sectores hiperbólicos y dos sectores parabólicos.

ii) Si $\widetilde{O}_{2}\left(0, k_{2}\right)$ es un punto silla, dos de cuyas separatrices se encuentran a ambos lados del eje $\eta$, entonces la vecindad de $O$ es la unión de seis sectores hiperbólicos.

iii) $\mathrm{Si} \widetilde{O}_{2}\left(0, k_{2}\right)$ es una silla nodo, y dos de sus regiones sillas se encuentran sobre un lado del eje $\eta$, la vecindad de $O$ es la unión de cuatro sectores hiperbólicos y un sector parabólico.

Lema 3. El sistema

$$
\left\{\begin{array}{l}
\frac{d x}{d t}=x y \\
\frac{d y}{d t}=-k y^{2}+x^{2} f(x, y)+x y f_{1}(x, y)
\end{array}\right.
$$

donde $f(x, y)$ y $f_{1}(x, y)$ son analíticas en la vecindad del origen, $k>0$ y $f(x, 0) \neq 0$, tiene exactamente dos semitrayectorias tendiendo al punto $O(0,0)$ en las direcciones $\theta=\pi / 2$ y $\theta=3 \pi / 2$, las cuales son el eje $y$ positivo y el eje negativo $y$.

Lema 4. Sean $f(x, y)$ y $\varphi(x)$ analíticas en la vecindad del $O(0,0), a \neq 0, b<0$ y $\varphi(0)=0$. Entonces el sistema

$$
\left\{\begin{array}{l}
\frac{d x}{d t}=x y, \\
\frac{d y}{d t}=a x[1+\varphi(x)]+b y^{2}+x y f(x, y)
\end{array}\right.
$$

tiene un punto silla en $O(0,0)$, cuyas separatrices son el positivo y negativo eje $y$, así como dos semitrayectorias las cuales también tienden a $O(0,0)$ en las direcciones $\theta=\pi / 2$ y $\theta=3 \pi / 2$; estos últimos se encuentran respectivamente en el primer y cuarto cuadrante si $a>0$, en el segundo y tercer cuadrante si $a<0$.

Definición 1. Un punto singular cuya vecindad es la unión de dos sectores parabólicos se llama punto singular degenerado. Si la vecindad del punto singular $O$ consiste de un sector hiperbólico y un sector elíptico, se dirá que $O$ es un punto singular con región eliptica.

\section{Parte Central}

Los siguientes diagramas son consecuencias del siguiente teorema, el cual es el resultado principal del presente artículo

Teorema 1. Para el sistema $\left(A_{0}\right)$ consideremos $k$ un número par, $k=2 m(m \geq 1)$. Entonces el punto singular $O(0,0)$ es

1. Un punto singular degenerado si $b_{n}=0, b_{n} \neq 0$ y $n \neq m$ (fig 1a);

2. Una silla-nodo si $b_{n} \neq 0$ y $n<m$ (fig $1 \mathrm{~b}$ ). 


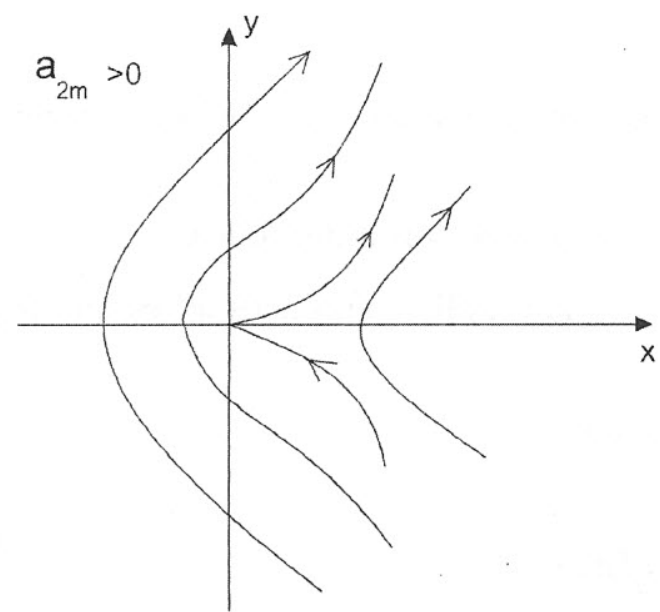

(a)

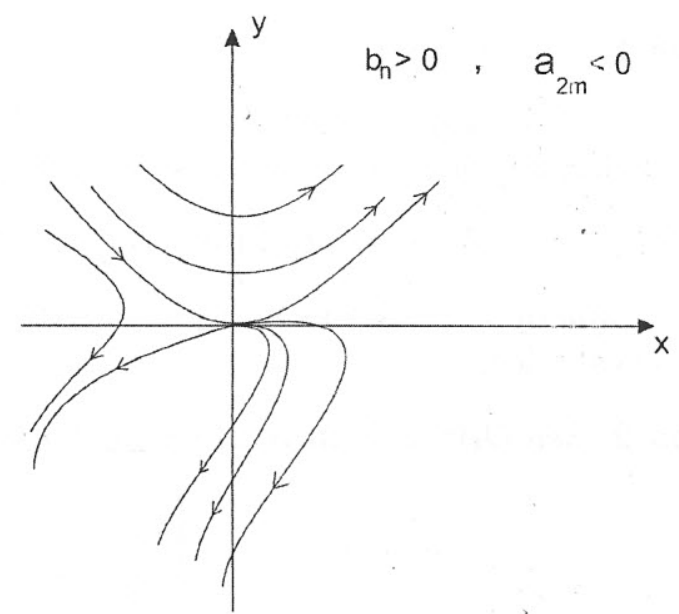

(b)

Figura 2:

\section{Prueba}

Si en la ecuación $\left(A_{r}\right)$ consideramos $k=2 m$ resulta

$$
\left\{\begin{array}{l}
\frac{d x}{d t}=\eta_{r} x, \\
\frac{d \eta_{r}}{d t}=-r \eta_{r}^{2}+a_{2 m} x^{2 m-2 r+1}[1+h(x)]+b_{n} x^{n-r+1} \eta_{r}[1+g(x)]+\eta_{r}^{2} x f\left(x, \eta_{r} x^{r}\right) .
\end{array}\right.
$$

Veamos el caso en que $b_{n}=0$ ó $b_{n} \neq 0$ y $n>m$.

Para todo $r$ tal que $1 \leq r \leq m-1$ se tiene que como $r<m$ entonces

$$
\begin{aligned}
r+1 \leq m & \Rightarrow \quad 2 r+2 \leq 2 m \\
& \Rightarrow \quad 2 m-2 r \geq 2 \\
& \Rightarrow \quad 2 m-2 r+1 \geq 3
\end{aligned}
$$

también se deduce que $r+1 \leq m<n \Rightarrow n-r+1>2$, luego las direcciones en las cuales las trayectorias de $\left(A_{r}\right)$ pueden acercarse a $O_{r}(0,0)$ son $0, \pi, \frac{\pi}{2}$ y $\frac{3}{2}$, pero por el lema hay dos trayectorias que tienden a $O_{r}(0,0)$ en las direcciones $\frac{\pi}{2}$ y $\frac{3}{2}$.

Para analizar las trayectorias que tienden a $O_{r}(0,0)$ en otras direcciones aplicamos una nueva explosión $x=x, \eta_{m-1}=\eta_{m} x, d \tau=x d t$ de donde obtenemos :

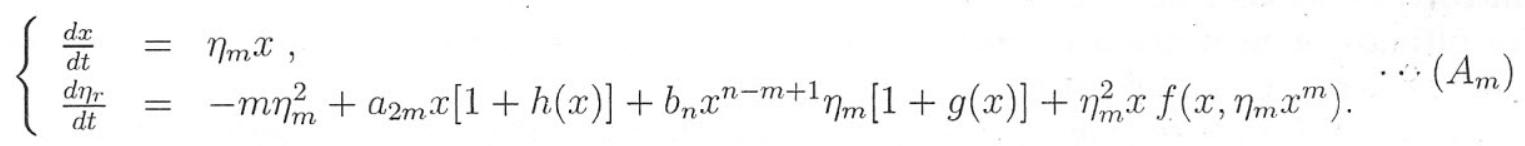

pero este sistema por lema lema 4 tiene un punto silla en $O_{m}(0,0)$.

Ahora, a cada trayectoria $L$ de $\left(A_{0}\right)$ la cual tiende a $O(0,0)$ en la dirección 0 ó $\pi$ le corresponde una trayectoria $L_{r}(r=0,1,2,3, \ldots, m)$ en el plano cortado el cual tiende a $O_{r}(0,0)$, pero como la dirección de aproximación a $O_{m}(0,0)$ es únicamente $\frac{\pi}{2}$ y $\frac{3 \pi}{2}$ esto indica que en el sistema $(A)$ hay solo dos trayectorias que tienden a $O$ en la dirección 0 y $\pi$.

Por tanto el punto singular $O(0,0)$ es un punto singular degenerado (ver figura 1 )

Ahora veamos el caso $r=n=m$ y $b_{n} \neq 0$.

En este caso el sistema $\left(A_{r}\right)$ es

$$
\left\{\begin{array}{l}
\frac{d x}{d t}=\eta_{m} x \\
\frac{d \eta_{m}}{d t}=-m \eta_{m}^{2}+a_{2 m} x[1+h(x)]+b_{n} x \eta_{m}[1+g(x)]+\eta_{m}^{2} x f\left(x, \eta_{m} x^{m}\right) . \cdots\left(A_{m}\right)
\end{array}\right.
$$


Las direcciones en las cuales las trayectorias de $\left(A_{r}\right)$ se acercan a $O_{m}(0,0)$ son $\pi / 2$ y $3 \pi / 2$, luego por el lema el punto $O_{m}(0,0)$ es punto silla y por tanto como el caso anterior $O(0,0)$ es punto silla degenerado.

Caso $b_{n} \neq 0$ y $n<m$.

Para $r=n$ se tiene el sistema:

$$
\left\{\begin{array}{l}
\frac{d x}{d t}=\eta_{n} x, \\
\frac{d \eta_{n}}{d t}=-n \eta_{n}^{2}+a_{2 m} x^{2 m-2 n+1}[1+h(x)]+b_{n} x \eta_{n}[1+g(x)]+\eta_{n}^{2} x f\left(x, \eta_{n} x^{n}\right) .
\end{array}\right.
$$

y como $n<m$ entonces $2 m-2 n \geq 2 \Rightarrow 2 m-2 n+1 \geq 3$.

Luego las direcciones de aproximación de las trayectorias del sistema $\left(A_{n}\right)$. son :

$$
\theta=\frac{\pi}{2}, \frac{3 \pi}{2}, 0, \pi, \arctan \left(\frac{b_{n}}{n+1}\right), \pi+\arctan \left(\frac{b_{n}}{n+1}\right) .
$$

Por el lema 3 hay sólo 2 trayectorias que tienden a $O_{n}(0,0)$ en las direcciones $\pi / 2$ y $3 \pi / 2$.

Analizaremos cuántas trayectorias existen acercándose a $O_{n}(0,0)$ en las otras direcciones, para lo cual al hacer una nueva explosión obtenemos el siguiente sistema al cual llamamos $\left(A_{n+1}\right)$.

$$
\left\{\begin{array}{l}
\frac{d x}{d t}=\eta_{n+1} x, \\
\frac{d \eta_{n+1}}{d t}=-(n+1) \eta_{n+1}^{2}+a_{2 m} x^{2 m-2 n+1}[1+h(x)]+b_{n} \eta_{n+1}[1+g(x)]+\eta_{n+1}^{2} x f\left(x, \eta_{n+1} x^{n+1}\right) .
\end{array}\right.
$$

Este sistema tiene singularidades $\widetilde{O}(0,0)$ y $\widetilde{O}_{1}\left(0, \frac{b_{n}}{n+1}\right)$.

Vemós que $2 m-2 n-1 \geq 1$ y entonces $\Delta\left(0, \frac{b_{n}}{n+1}\right)<0$ lo cual nos dice que $\widetilde{O}_{1}$ es un punto silla.

Para $O(0,0)$ tenemos que

$$
\triangle=\left|\begin{array}{cc}
0 & 0 \\
a_{2 m} & b_{n}
\end{array}\right|=0
$$

de donde tenemos un $\lambda=b_{n}$, ahora haciendo el cambio $b_{n} d t=d \tau$ en $\left(A_{n+1}\right)$ se obtiene

$$
\left\{\begin{array}{l}
\frac{d x}{d \tau}=\frac{\eta_{n+1} x}{b_{n}}, \\
\frac{d \eta_{n+1}}{d \tau}=-(n+1) \eta_{n+1}^{2}+\frac{a_{2 m}}{b_{n}} x^{2 m-2 n+1}[1+h(x)]+\eta_{n+1}[1+g(x)]+\frac{\eta_{n+1}^{2}}{b_{n}} x f\left(x, \eta_{n+1} x^{n+1}\right) .
\end{array}\right.
$$

ahora hacemos $\eta_{n+1}=y, \mathrm{y}, d \tau=d t$ y obtenemos

$$
\left\{\begin{array}{l}
\frac{d x}{d t}=\frac{x y}{b_{n}}, \\
\frac{d y}{d t}=-\frac{(n+1)}{b_{n}} y^{2}+\frac{a_{2 m}}{b_{n}} x^{2 m-2 n+1}[1+h(x)]+y[1+g(x)]+\frac{y^{2}}{b_{n}} x f\left(x, y x^{n+1}\right) .
\end{array} \cdots\left(A_{n+1}^{\prime}\right)\right.
$$

Sea

$$
Q(x, y)=-\frac{(n+1)}{b_{n}} y^{2}+\frac{a_{2 m}}{b_{n}} x^{2 m-2 n+1}[1+h(x)]+\frac{y^{2}}{b_{n}} x f\left(x, y x^{n+1}\right)
$$

Ahora si consideramos una polinomial general

$$
y=\phi(x)=a_{0}+a_{1} x+a_{2} x^{2}+\cdots
$$

podemos hallar los $a_{j}$ al reemplazar en $y+Q(x, y) \equiv 0$, es decir $\left(a_{0}+a_{1} x+a_{2} x^{2}+\cdots\right)+\frac{a_{2 m}}{b_{n}} x^{2 m-2 n-1}+x \frac{\phi^{2}(x)}{b_{n}} f\left(x, x^{n+1} \phi(x)\right)-\frac{(n+1)}{b_{n}}\left(a_{0}+a_{1} x+\cdots\right)^{2} \equiv 0$ resultando que $a_{0}=0$. Como $2 m-2 n-1 \geq 1$ entonces

$$
\left(a_{1}+\frac{a_{2 m}}{b_{n}}-\frac{(n+1)}{b_{n}}\left(2 a_{0} a_{1}\right)\right) x \equiv 0 \Rightarrow a_{1}=-\frac{a_{2 m}}{b_{n}}
$$


por tanto

$$
y=\phi(x)=-\frac{a_{2 m}}{b_{n}} x+\cdots
$$

si lo reemplazamos en la primera ecuación de $\left(A_{n+1}^{\prime}\right)$ se tiene

$$
\Psi(x)=\left(-\frac{a_{2 m}}{b_{n}} x+\cdots\right) \frac{x}{b_{n}}=-\frac{a_{2 m}}{b_{n}} x^{2}+\cdots
$$

es decir

$$
\Psi(x)=A_{m} x^{m}+\cdots
$$

donde $m=2, A_{m}>0$ siempre y cuando $a_{2 m}<0$.
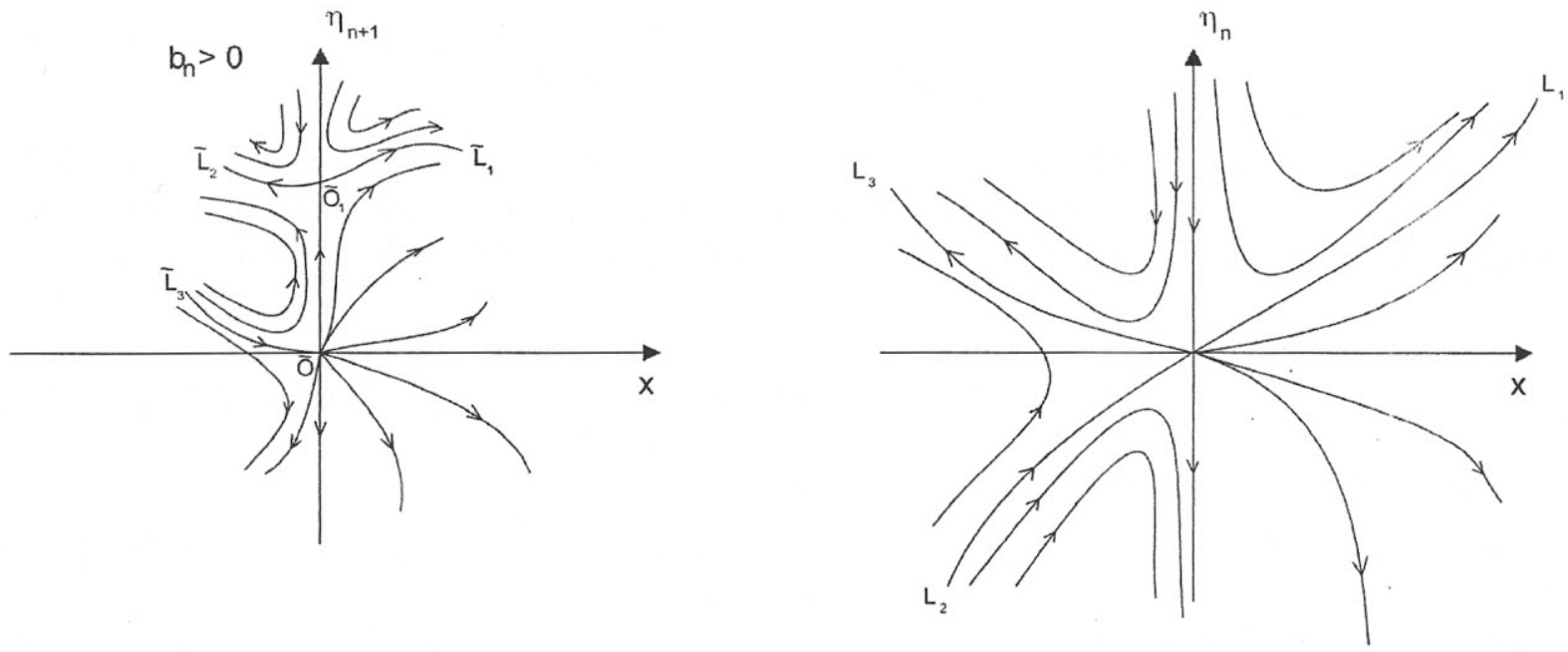

Figura 3:

Esto indica que $\widetilde{O}(0,0)$ es una silla-nodo.

Luego el sistema $\left(A_{n+1}\right)$ tiene la configuración dada en la figura 2.

La última configuración la tienen $\operatorname{los}\left(A_{r}\right), r=1,2, \ldots, n$; finalmente para obtener el plano de fase de $\left(A_{0}\right)$ usamos la transformación

$$
T(x, \eta)=(x, \eta x)
$$

con lo cual la configuración resultante es una silla-nodo, con lo cual se concluye el teorema.

Conclusiones El resultado probado completa el estudio del sistema

$$
\left\{\begin{array}{l}
\frac{d x}{d t}=y, \\
\frac{d y}{d t}=a_{k} x^{k}[1+h(x)]+b_{n} x^{n} y[1+g(x)]+y^{2} f(x, y),
\end{array}\right.
$$

y su comportamiento alrededor de un punto singular aislado. 


\section{REFERENCIAS BIBLIOGRÁFICAS}

[1] Andronov, A. A. and Leontovich (1973). Qualitative Theory of Second-Order Dynamic Systems. Israel Program for Scientific Translations, Jerusalem, Wiley, New York.

[2] Perko, L. (1991). Differential Equations and Dynamical Systems. Springer-Verlag.

[3] Sotomayor, J. (1979). Liçoes de Equaçóes Diferenciais Ordinárias. Projeto Euclides, IMPA.

[4] Sulca, T. (1997).Tesis de Licenciatura. UNMSM Lima-Perú.

[5] Sulca, T. (2007). Caso de un sistema no hiperbólico. PESQUIMAT Vol.X , $N^{\circ} 1$ : $45-54$.

[6] Zhang zhi-Fen (1992). Qualitative theory of differential equations. Translations of Mathematical Monographs, Vol 101. Printed by The American Mathematical Society. 